\title{
Allocution d'ouverture
}

Monsieur le Préfet de réGION,

Monsieur le Conseiller d'Etat,

Monsieur Le Recteur,

Messieurs les Professeurs,

Mesdames, Messieurs,

Jusqu'au dernier moment, le Délégué général Aigrain comptait assister à cette journée en ouvrant le colloque de Montpellier.

Mais ses charges universitaires, qui le retiennent à Paris aujourd'hui, ne lui ont pas permis d'effectuer le déplacement qu'il avait envisagé et qu’il avait souhaité vivement.

En le quittant vendredi, il m'a demandé de vous exprimer ses regrets et de vous dire, à sa place, les quelques mots qui s'imposent en pareille circonstance.

La D.G.R.S.T., organe de coordination de la recherche scientifique française, a lancé, en 1965, au début de la période couverte par le $V^{*}$ Plan, une action concertée Lutte Biologique en vue de développer ce secteur de recherche.

A cette fin, un Comité scientifique d'action concertée, doté d'un financement spécial, a été mis en place de manière à apporter aux laboratoires compétents un complément de moyens d'investissement et de fonctionnement. Si une telle entreprise a pu être envisagée par le Gouvernement français, c'est parce que, depuis une quinzaine d'années, diverses institutions scientifiques françaises et plus particulièrement l'Institut National de la Recherche agronomique ou encore l'Institut Pasteur avaient déjà consenti à faire d'importants efforts dans cette voie.

Le colloque de Montpellier sur la Lutte Biologique contre les arthropodes hématophages et la pathologie des vecteurs s'inscrit dans ce cadre.

La lutte contre les Arthropodes vecteurs de germes pathogènes pour l'homme, les animaux ou les végétaux constitue une tâche dont l'importance au plan national et mondial n'est plus à souligner. Les progrès dans ce domaine de recherche intéressent directement la collectivité humaine. En médecine d'une part, la transmission des protozooses et des viroses par des insectes piqueurs et la dissémination mécanique par de nombreux Arthropodes constituent un problème pour la santé publique, problème 
particulièrement aigu en zone tropicale. En agriculture d'autre part, les vecteurs de maladies ont un rôle déterminant dans le domaine vétérinaire et constituent un danger permanent pour la protection sanitaire des cultures et des forêts.

La lutte contre les vecteurs se heurte à des difficultés semblables à celles déjà rencontrées dans le domaine des autres ravageurs de cultures. En effet, tout comme au cours des traitements chimiques contre les insectes nuisibles aux cultures, de nombreux échecs ont été enregistrés et les problèmes de l'installation de résistance, du danger de l'usage abusif d'insecticides de plus en plus toxiques et de l'inefficacité liée aux motifs d'ordre écologique, existent avec acuité et suggèrent l'introduction d'autres méthodes. La rémanence de certains résidus et leur présence dans la chaîne trophique constituent une grave préoccupation pour l'homme.

Alors qu'en agriculture, l'emploi de méthodes biologiques contre les insectes a été envisagé depuis fort longtemps, un tel principe n'a été soulevé que depuis peu dans le domaine des vecteurs. Le Comité spécialisé de l'organisation mondiale de la santé, réuni à Gainsville (U.S.A.), en 1963, a mis en évidence la nécessité d'intensifier les travaux de recherche dans le domaine de la Lutte Biologique contre les insectes vecteurs, soulignant au passage le grand retard des recherches de base et la rareté des exemples de réalisations concrètes.

Pour ces raisons, le Comité scientifique Lutte biologique de la D.G.R.S.T. a voulu renforcer ce secteur de recherche en s'appuyant sur deux laboratoires pionniers situé dans la région :

- le laboratoire d'écologie médicale et de pathologie parasitaire de la Faculté de médecine de Montpellier dirigé par le Professeur Rioux ;

- le laboratoire de cythopathologie de l'I.N.R.A.-C.N.R.S. dirigé par le professeur Vago : ce laboratoire est installé à Saint-Christol, près de Montpellier, et, à Montpellier même, dans les locaux de la Faculté des Sciences.

Nous sommes réunis ici, pour parler de ces problèmes et faire le point des travaux entrepris depuis quatre ans, ce n'est pas la seule raison du choix de Montpellier.

En plaçant le colloque sous la présidence du Conseiller d'Etat Racine, Président de la Mission interministérielle pour l'aménagement du Languedoc-Roussillon qui vient de recevoir récemment du Gouvernement de hautes et nouvelles responsabilités en étant nommé à la direction de l'Ecole Nationale d'Administration, en choisissant Montpellier comme lieu du déroulement de ce colloque, le groupe de travail chargé de l'organisation a souhaité établir un dialogue entre la recherche et les utilisateurs de ses résultats scientifiques au sein même d'une région, en cours d'aménagement, où les arthropodes vulnérants sont particulièrment abondants et peuvent zonstituer un frein à son développement économique et touristique. 
Monsieur le Préfet de région, votre présence à ce colloque témoigne de l'intérêt que porte le Gouvernement au développement de la recherche scientifique mise au service de l'aménagement du territoire et de l'hygiène publique. Elie constitue une incitation de nos efforts et nous honore particulièrement. Nous vous en sommes très vivement reconnaissants.

Monsieur le Conseiller d'Etat, à la tête de la mission interministérielle LanguedocRoussillon, vous avez en charge la responsabilité de la coordination des diverses administrations qui interviennent dans l'aménagement touristique du littoral. Cette tâche que vous exercez avec une compétence éclectique vous conduit à ne pas négliger les solutions que la recherche scientifique est susceptible d'apporter à court et à moyen terme. Vous avez pris une part active aux travaux du groupe préparatoire et vos avis, très appréciés, ont été un grand encouragement pour la réalisation de ces journées.

Monsieur le Recteur, malgré vos lourdes charges en cette période de rentrée universitaire, vous avez tenu à montrer, par votre présence, le grand intérêt que le Ministre de l'Education Nationale au développement de la recherche et de ses applications.

Permettez-moi de saluer, au nom du Délégué général :

M. Grison, Président du Comité,

M. L'Héritier, Professeur à la Faculté des Sciences de Clermont-Ferrand, généticien de grand renom qui participa, il y a quelques années aux travaux du Comité scientifique «Applications de la Génétique » et qui fut responsable en particulier de la Commission de formation où son rôle fut très actif et efficace.

M. Biliotti, Chef de département de zoologie de l'I.N.R.A. et Président de l'organisation internationale de Lutte Biologique.

Je n'aurai garde d'oublier nos invités étrangers que je remercie d'avoir accepté d'effectuer des communications scientifiques sur l'état d'avancement de leurs travaux. Certains d'entre eux sont venus de fort loin pour apporter à Montpellier le fruit de leurs recherches et de leur expérience en la matière.

M. le Professeur Coluzzi de l'Institut de Parasitologie de la Faculté des sciences de Rome dont les travaux sur l'hétérochromosome d'Anopheles gambiae sont considérés comme un modèle du genre.

M. le Professeur Graig de l'Université américaine de Notre-Dame dans l'Etat d'Indiana qui est le leader mondial de la lutte génétique contre les Culicides.

M. le Professeur Davidson de l'Institut d'hygiène tropicale de Londres qui a récemment réalisé plusieurs tentatives de lutte génétique par l'emploi de mâles stériles.

M. le Professeur Kurstak de l'Université de Montréal, spécialiste de l'utilisation des virus d'insectes en lutte biologique qui poursuit des travaux sur la densonucléose des lépidoptères en liaison avec le laboratoire de Saint-Christol.

M. le Professeur Laven de l'Université de Mayence, généticien allemand de renommée mondiale qui a démontré pour la première fois l'existence des phénomènes d'incompatibilité cytoplasmique et dont l'expérience de Rangoon sur Culex fatigans, réalisée avec le soutien de l'O.M.S. a eu un grand retentissement scientifique. 


\section{J. DAGUIN}

Le docteur Pal de l'organisation mondiale de la santé, responsable auprès de cette organisation des programmes de lutte génétique. Ses travaux effectués aux Indes sur les Culex et sur les Anophèles ont porté sur la génétique de la résistance aux insecticides. Sa contribution au remarquable ouvrage sur \&la génétique des insectes vecteurs de maladies» a été déterminante.

M. le Professeur Peters du centre agronomique de Wageningen dont les principaux travaux ont porté sur la transmission des virus des végétaux par les Aphides; il fut le premier à isoler le virus de l'enroulement, maladie très importante du point de vue économique.

M. le Professeur Rehacek qui dirige le laboratoire de virologie de l'Académie des sciences de Bratislava, spécialiste des cultures de tissus d'insectes vecteurs. Ses travaux portent surtout sur les virus et les rickettsies transmis par les tiques.

Je remercie également M. Boesiger du C.N.R.S., M. Morel de l'I.E.M.V.T. et M. Mouchet de l'O.R.S.T.O.M., de leur contribution à nos journées.

Enfin, mes remerciements iront vers le professeur Rioux et ses collaborateurs qui ont eu la lourde charge de veiller à l'organisation du colloque et de régler dans leurs moindres détails les diverses questions matérielles qui ne manquent pas de se poser en pareille occasion.

Je déclare ouvert le colloque de Montpellier sur la Lutte Biologique contre les arthropodes hématophages et la pathologie des vecteurs et je prie M. le Conseiller d'Etat Racine de bien vouloir assurer la présidence de ces journées au cours desquelles il sera successivement assisté de MM. Biliotti, Boesiger, Grison et Vago.

\section{J. DAGUIN,}

Secrétaire scientifique de la D.G.R.S.T. 